$\xi=-1$

\title{
A Simulated Kalman Filter Optimizer with White Hole Operator
}

\author{
Suad Khairi Mohammed ${ }^{1}$, Badaruddin Muhammad ${ }^{2}$, Nor Azlina Ab. Aziz ${ }^{3}$, Nor Hidayati Abdul Aziz, \\ Tasiransurini Ab Rahman ${ }^{4}$, Norazian Subari², Mohd Saberi Mohamad ${ }^{5}$ and Zuwairie Ibrahim ${ }^{6 *}$ \\ ${ }^{1}$ Department of Electrical Engineering, University of Technology, Baghdad, Iraq. \\ ${ }^{2}$ Faculty of Electrical and Electronics Engineering, Universiti Malaysia Pahang, 26600 Pekan, Pahang, Malaysia. \\ ${ }^{3}$ Faculty of Engineering and Technology, Multimedia University, 75450 Bukit Beruang, Melaka, Malaysia. \\ ${ }^{4}$ Faculty of Electrical and Electronic, Universiti Tun Hussein Onn Malaysia, 86400 Johor, Malaysia. \\ ${ }^{5}$ Institute of Artificial Intelligence and Big Data, Faculty of Bioengineering and Technology, Universiti Malaysia Kelantan, Malaysia. \\ ${ }^{6}$ Faculty of Manufacturing Engineering, Universiti Malaysia Pahang, 26600 Pekan, Pahang, Malaysia. \\ *Corresponding author E-mail: zuwairie@ump.edu.my
}

\begin{abstract}
The simulated Kalman filter (SKF) is a population-based optimization algorithm that was developed based on a well-known estimator called Kalman filter. Meanwhile, a white hole operator has been recently introduced to prevent premature convergence in black hole algorithm (BHA). The computation of white hole operator begins by selecting the worst agent as the white hole with event horizon. If an agent is located within the event horizon of white hole, the agent is pushed by the white hole. In this study, the white hole operator is used to improve the effectiveness of the SKF optimizer. A comprehensive experiment is done to evaluate the proposed SKF with white hole operator (SKFWH).
\end{abstract}

Keywords: Optimization; Simulated Kalman filter; White hole.

\section{Introduction}

Recently, the use of a white hole operator to improve a black hole algorithm has been reported in literature [1-2]. The white hole operator was proposed to avoid the agents from exploring the area near the worst agent, which is also called the white hole agent. If an agent is located near the white hole agent, that agent is pushed away by the white hole agent.

On the other hand, the simulated Kalman filter (SKF) algorithm [34] is inspired by the estimation capability of Kalman filtering [5]. In SKF algorithm, every agent is regarded as a Kalman filter. Based on the mechanism of Kalman filtering and measurement process, every agent estimates the global minimum/maximum in a search space. To date, the SKF algorithm has been applied to solve several engineering problems. In signal processing, Adam et al. has employed angle-modulated SKF as feature selection in peak classification of EEG signal [6-7]. In telecommunication engineering, the SKF algorithm has been used as adaptive beamforming algorithm [8-11]. In industrial engineering, the SKF has been used to solve printed circuit board drill path optimization problem [12-13] and assembly sequence planning problem [14]. In scheduling application, the SKF has been employed in solving airport gate allocation problem [15-16]. In image processing, the SKF has been used as a template matching algorithm in distance measurement [17-18]. In system identification, the SKF algorithm has been used to estimate the model order and parameter value of an ARX model [1920]. The SKF algorithm also has been introduced as a tuning method for proportional-integral-derivative (PID) controller [21]. Fundamentally, studies of the SKF algorithm have been reported
[22-23]. Furthermore, modifications of the SKF [24-25] and hybridization with other algorithms [26-30] have been done to further improve the performance of the SKF algorithm. Several extensions of SKF algorithm for combinatorial optimization problems have also been introduced [31-34].

This paper presents a new improvement to SKF optimizer using the recently introduced white hole operator [35]. The effectiveness of the SKF optimizer with white hole operator is evaluated based on unimodal, multimodal, hybrid, and composite functions in CEC2014 benchmark test functions. Results show that the used of white hole operator significantly improves the SKF as global optimization algorithm.

\section{The simulated Kalman filter optimizer}

The simulated Kalman filter (SKF) algorithm is illustrated in Figure 1. Consider $n$ number of agents, SKF algorithm begins with initialization of $n$ agents randomly. The maximum number of iterations, $t_{\max }$, the initial value of error covariance estimate, $P(0)$, the process noise covariance value, $Q \in[0,1]$, and the measurement noise covariance value, $R \in[0,1]$, are defined during the initialization stage. After the initialization, every agent is subjected to fitness evaluation to produce initial solutions $\left\{\boldsymbol{X}_{1}(0), \boldsymbol{X}_{2}(0), \boldsymbol{X}_{3}(0), \ldots, \boldsymbol{X}_{n-2}(0), \boldsymbol{X}_{n-1}(0)\right.$, $\left.\boldsymbol{X}_{n}(0)\right\}$. The fitness values are compared and the agent having the best fitness value at every iteration, $t$, is registered as $\boldsymbol{X}_{\text {best }}(t)$. Subsequently, $\boldsymbol{X}_{\text {true }}$ is updated only if the $\boldsymbol{X}_{\text {best }}(t)$ is better than the $\boldsymbol{X}_{\text {true }}$. As shown in Figure 1, there are 6 important computations in the SKF algorithm. 


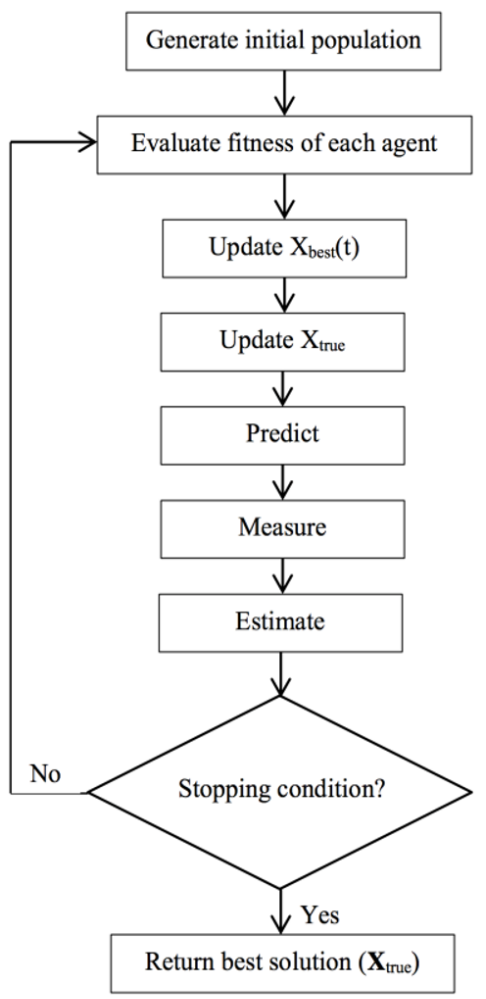

$$
\begin{aligned}
& \text { Prediction } \\
& \boldsymbol{X}_{i}(t \mid t+1)=\boldsymbol{X}_{i}(t) \\
& \boldsymbol{P}(t \mid t+1)=\boldsymbol{P}_{i}(t)+Q
\end{aligned}
$$

where $\boldsymbol{X}_{i}(t)$ and $\boldsymbol{X}_{i}(t \mid t+1)$ denote the current state and current transition/predicted state, respectively, while the $\boldsymbol{P}_{i}(t)$ and $\boldsymbol{P}(t \mid t+1)$ denote the current error covariant estimate and current transition error covariant estimate, respectively.

\section{Measurement}

$\boldsymbol{Z}_{i}(t)=\boldsymbol{X}_{i}(t \mid t+1)+\sin \left(2 \pi r_{i}\right) \times\left|\boldsymbol{X}_{i}(t \mid t+1)-\boldsymbol{X}_{\text {true }}\right|$

\section{Estimate}

$K(t)=\frac{P(t \mid t+1)}{P(t \mid t+1)+R}$

Then, the estimation of next state, $\boldsymbol{X}_{i}(t+1)$, and the updated error covariant, $P_{i}(t+1)$, are computed based on (5) and (6), respectively.

$$
\begin{aligned}
& \boldsymbol{X}_{i}(t+1)=\boldsymbol{X}_{i}(t \mid t+1)+K(t) \times\left|\boldsymbol{Z}_{i}(t)-\boldsymbol{X}_{i}(t \mid t+1)\right| \\
& P(t+1)=(1-K(t)) \times P(t \mid t+1)
\end{aligned}
$$

Fig. 1: The original simulated Kalman filter algorithm [3-4].

\section{The white hole operator}

If the black holes exist, then it should be possible to reverse the equations governing them to get the opposite of black hole, which is the white hole. As oppose to black hole agent in the black hole algorithm (BHA) [36], the white hole can be assigned to the worst agent in the population.

The white hole has its own event horizon as shown in Figure 2. The radius of the event horizon, $R_{W H}$, can be calculated based on the following equation:

$R_{W H}=\frac{f i t_{W H}}{\sum_{i=1}^{N} f i t_{i}}$

where fitwH is the fitness value of the white hole, $N$ is the number of agents, and $f i t_{i}$ is the fitness value of the $i^{\text {th }}$ agent.

As shown in Figure 2(a), an arbitrary agent $i$ may be positioned within the event horizon of the white hole. In this case, the agent is pushed by the white hole as illustrated in Figure 2(b). Due to this, the position of the agent $i$ is updated as follows:

$X_{i}(t+1)=X_{i}(t)+\operatorname{rand} \times\left(X_{W H}+X_{i}(t)\right)$

where $X_{i}(t+1)$ and $X_{i}(t)$ are the locations of the arbitrary agent $i$ at iterations $t+1$ and $t$, respectively. The rand is a random number belonging to $[0,1]$ and $X_{W H}$ is the location of the white hole agent. This white hole operator is computed after the measurement step in SKF algorithm as shown in Figure 3. Note that the white hole operator is applied only to the agent that is located within the event horizon of a white hole agent. Otherwise, the solution is updated using (5) as of the original SKF algorithm.

\section{Experiment, Result, and Discussion}

The CEC2014 Benchmark Test Suite for single-objective optimization [37] was employed to observe the performance of the SKF algorithm with white hole operator. This test suite comprises of 30 functions of minimization problem. The experiments were repeated for several runs and the mean fitness was calculated. In all experiments, $Q=R=0.5$.

In the first experiment, the performance of the proposed SKFWH algorithm is compared with the performance of the original SKF algorithm. Using the CEC2014 functions, the problems' dimension can be adjusted. Therefore, 10-dimensions and 50-dimenstions test functions were tested. Obviously, 50-dimensions test functions are more difficult to solve than the 10-dimensions test functions. The optimization process involved 100 agents and it is stopped at $1,000,000^{\text {th }}$ function evaluation. After 50 runs, the mean fitness was calculated as tabulated in Table 1 and Table 2.

To analyze the experimental result, Wilcoxon signed rank test [38] was used for pairwise non-parametric statistical analysis. The Wilcoxon test usually is used when the population cannot be assumed to be normally distributed or it can be used to compare two related samples, matched samples, or repeated measurements on a single sample to assess whether their population mean ranks differ. The null hypothesis for the test assumes that there is no significant difference between the mean values of test algorithm and competing algorithm while the alternative hypothesis tries to determine if there is a significant difference between those two algorithms using 5\% $(\alpha=0.5)$ significance level. Since the number of samples is 30 , the critical value for the test is equal to 137 . The sum of ranks where the test algorithm outperforms a competing algorithm is denoted as $\mathrm{R}+$ while the sum of ranks where the test algorithm is outperformed by the competing algorithm is denoted as R-. Hence, the test algorithm is better than the competing algorithm if $\mathrm{R}+>\mathrm{R}$ - and the competing algorithm is significantly better than the $\mathrm{BH}$ algorithm if $\mathrm{R}$ - value is less than the critical value. 


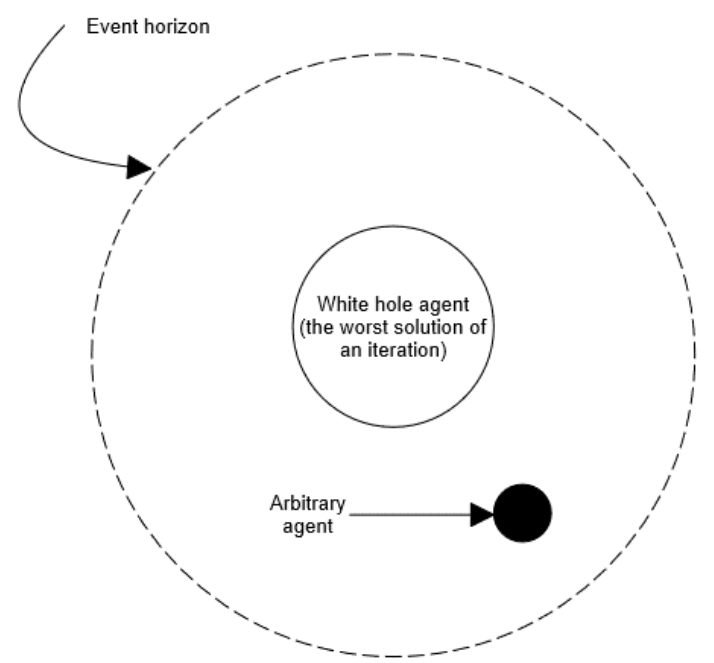

(a)

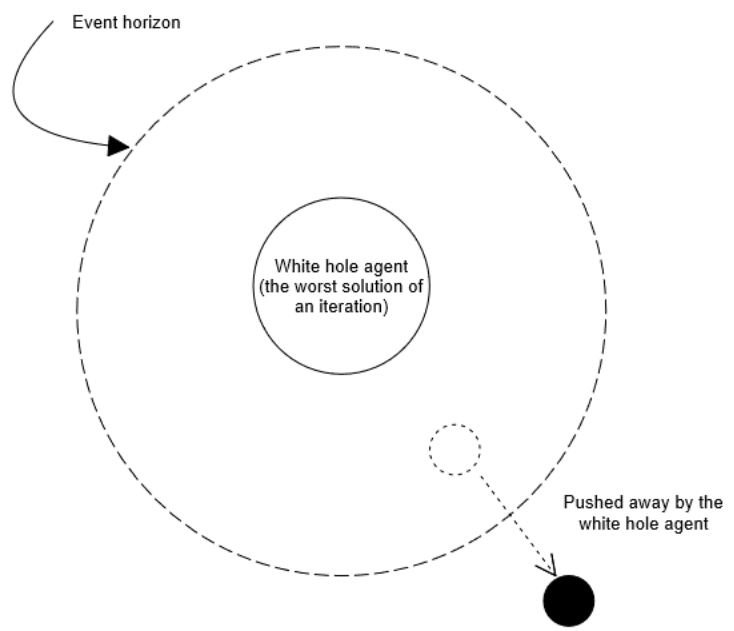

(b)

Fig. 2: The concept of white hole operator (a) The white hole agent (the worst solution of an iteration), the white hole's event horizon, and an arbitrary agent (located within the event horizon) are shown. (b) The agent located within the event horizon is pushed away by the white hole agent.

Based on Table 1 and Table 2, for the 10-dimensions problem, out of 30 functions, the SKFWH outperforms the original SKF in 27 test functions, while for the 50-dimensions problem, the SKFWH outperforms the original SKF in 21 test functions. Based on the Wilcoxon signed rank test results shown in Table 3 and Table 4, since $\mathrm{R}-<137$ ( $\mathrm{R}$ - value is written in bold if it is less than 137), the proposed SKFWH outperforms the original SKF for both cases. This analysis indicates that the proposed SKFWH would performs significantly better than the original SKF in solving low and high dimensional problems.

For the second experiment, the number of agents of SKFWH were increased from 10 to 50 and finally to 100 while maintaining the number of function evaluations $(N F E)$ as $1,000,000$. The number of function evaluations can be calculated as $N F E=$ number of agents $\times$ number of iterations. The number of dimensions in this experiment is 50. The mean fitness values are tabulated in Table 5. Based on the result of the Wilcoxon signed rank test in Table 6 , since the critical value is 137 , SKFWH with 50 agents performs significantly better than the SKFWH with 100 agents. R- value is written in bold if it is less than 137.

The third experiment investigates the importance of different initial values of error covariance estimate, $P(0)$, towards the performance of the SKFWH algorithm. The number of agents is 100 , the number of iterations is 10,000 , and the number of dimensions is 50 . Several $P(0)$ values were investigated. Those values are $1,10,100$, and
1,000. The mean fitness values are tabulated in Table 7. Based on the mean fitness values, the Wilcoxon signed ranked test was performed and the results are tabulated in Table 8 . R- value is written in bold if it is less than 137. It is found that none of the pairwise tests show any significant difference. Hence, the initial values of error covariance estimate, $P(0)$, gives no impact to the performance of the SKFWH algorithm.

The last experiment investigates the performance of the SKFWH algorithm with different values of the number of agents, which are 10,100 , and 1000 agents. In this experiment, the number of iterations is 100 and the number of dimensions is 50 . The mean fitness values are tabulated in Table 9. Based on the mean fitness values, the Wilcoxon signed ranked test was performed and the results are tabulated in Table 10. $\mathrm{R}$ - value is written in bold if it is less than 137. It is obvious that when the number of function evaluation is no longer a limit, significantly better results could be obtained when the number of agents is increased.

\section{Conclusion}

A lot of improvements of the SKF algorithms were reported in literature. However, this is the first time the SKF algorithm is improved using a new white hole operator. This white hole operator has been originally used to improve the black hole algorithm. In 
future, the usefulness of the white hole operator will be further investigated and tested to other optimization algorithm, such as finite impulse response optimizer [39].

\section{Acknowledgement}

The first author is heartily thankful to the Universiti Malaysia Pahang (UMP) for the internal research fund (RDU1703224) provided to perform this research. All the authors are grateful to the reviewers who made good efforts in reviewing this paper.

Table 1: The mean fitness based on 10-dimension test functions. Values in bold indicate smaller mean fitness.

\begin{tabular}{|c|c|c|c|}
\hline Types & No. & SKFWH & SKF \\
\hline \multirow[b]{2}{*}{ Unimodal functions } & 1 & \begin{tabular}{|l|l|}
3912948.948 \\
\end{tabular} & 5607771.528 \\
\hline & 2 & 1885.564735 & 2672632.715 \\
\hline \multirow{14}{*}{$\begin{array}{l}\text { Simple } \\
\text { multimodal functions }\end{array}$} & 3 & 1718.212752 & 3448.011551 \\
\hline & 4 & 420.3010925 & 420.91571 \\
\hline & 5 & 519.9999529 & 520.0063855 \\
\hline & 6 & 602.4705525 & 601.9204931 \\
\hline & 7 & 700.1624849 & 1139329685566 \\
\hline & 8 & 800 & 802.5417592 \\
\hline & 9 & 909.9052372 & 913.3856203 \\
\hline & 10 & 1000.553511 & 1121.796832 \\
\hline & 11 & 1405.400471 & 1598.084375 \\
\hline & 12 & 1200.080214 & 1200.122715 \\
\hline & 13 & 1300.241882 & 1300.265143 \\
\hline & 14 & 1400.165654 & 1400.329805 \\
\hline & 15 & 1501.204535 & 1501.436656 \\
\hline & 16 & 1602.286956 & 1602.519754 \\
\hline \multirow{6}{*}{$\begin{array}{l}\text { Hybrid } \\
\text { functions }\end{array}$} & 17 & 137217.0239 & 238934.7477 \\
\hline & 18 & 10378.26401 & 10225.36109 \\
\hline & 19 & 1901.015338 & 1901.262963 \\
\hline & 20 & 4420.818952 & 7589.154424 \\
\hline & 21 & 4894.618402 & 45650.08519 \\
\hline & 22 & 2211.437594 & 2264.463448 \\
\hline \multirow{8}{*}{ Composition functions } & 23 & 2622.868598 & 2629.807706 \\
\hline & 24 & 2523.171623 & 2525.825028 \\
\hline & 25 & 2670.516412 & 2688.776931 \\
\hline & 26 & 2700.154528 & 2700.189843 \\
\hline & 27 & 2772.816517 & 2948.974324 \\
\hline & 28 & 3283.214765 & 3308.24095 \\
\hline & 29 & 3253.417745 & 72370.09974 \\
\hline & 30 & 4247.948502 & 4241.064993 \\
\hline
\end{tabular}

Table 2: The mean fitness based on 50-dimension test functions. Values in bold indicate smaller mean fitness.

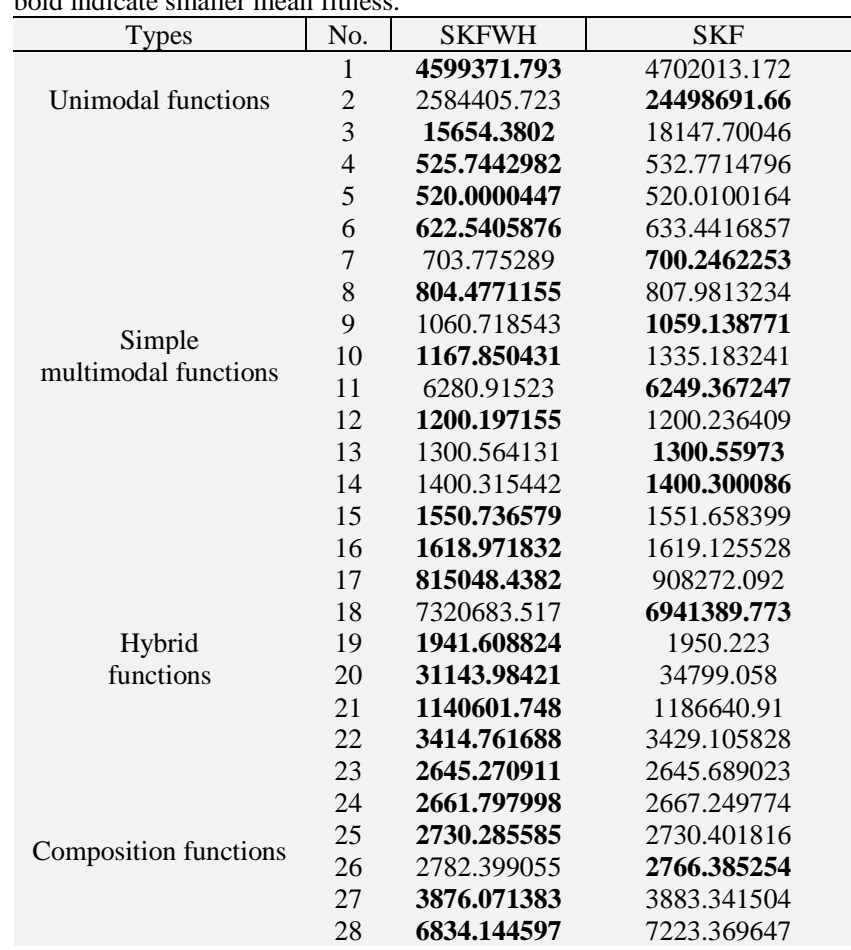

\begin{tabular}{lll}
29 & 8049.657217 & $\mathbf{5 9 9 7 . 8 3 0 1 6 6}$ \\
30 & $\mathbf{1 9 7 0 8 . 4 7 7 0 1}$ & 19753.28876 \\
\hline
\end{tabular}

Table 3: Wilcoxon signed rank test result based on 10-dimension test functions.

\begin{tabular}{l|l|l}
\hline & $\mathrm{R}+$ & $\mathrm{R}-$ \\
\hline SKFWH vs SKF & 422 & $\mathbf{4 3}$
\end{tabular}

Table 4: Wilcoxon signed rank test result based on 50-dimension test functions.

\begin{tabular}{c|c|c}
\hline & $\mathrm{R}+$ & $\mathrm{R}-$ \\
\hline SKFWH vs SKF & 352 & $\mathbf{1 1 3}$
\end{tabular}

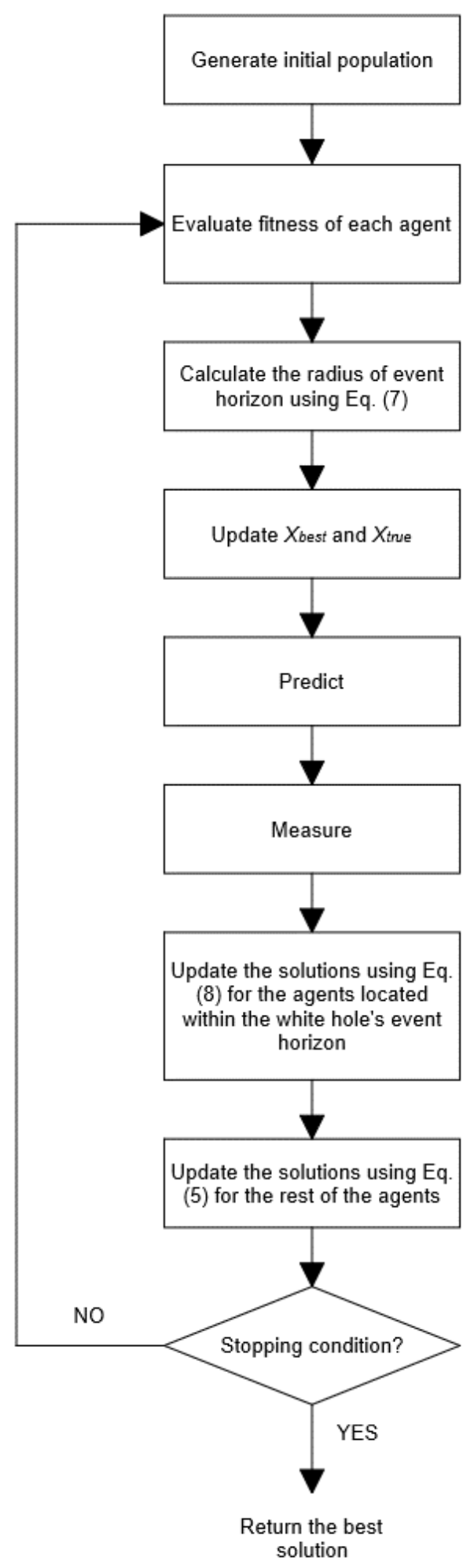

Fig. 3: The SKF algorithm with white hole operator (SKFWH). 
Table 5: The mean fitness based on 10, 50, and 100 agents. The total number of function evaluation is $1,000,000$

\begin{tabular}{|c|c|c|c|}
\hline No. & 10 agents & 50 agents & 100 agents \\
\hline 1 & 6842461.074 & 8249749.934 & 4599371.793 \\
\hline 2 & 7557.765436 & 67721025.25 & 2584405.723 \\
\hline 3 & 7180.761822 & 20435.18084 & 15654.3802 \\
\hline 4 & 558.8601389 & 542.2949562 & 525.7442982 \\
\hline 5 & 520.0008579 & 520.0000267 & 520.0000447 \\
\hline 6 & 634.3263996 & 626.2770105 & 622.5405876 \\
\hline 7 & 700.0483049 & 700.2555538 & 703.775289 \\
\hline 8 & 801.6259361 & 805.221172 & 804.4771155 \\
\hline 9 & 1110.712509 & 1072.622085 & 1060.718543 \\
\hline 10 & 1021.795305 & 1164.888077 & 1167.850431 \\
\hline 11 & 6629.145251 & 6370.193368 & 6280.91523 \\
\hline 12 & 1200.248523 & 1200.206515 & 1200.197155 \\
\hline 13 & 1300.548525 & 1300.564763 & 1300.564131 \\
\hline 14 & 1400.314432 & 1400.310443 & 1400.315442 \\
\hline 15 & 1574.222179 & 1578.482811 & 1550.736579 \\
\hline 16 & 1619.069307 & 1619.797243 & 1618.971832 \\
\hline 17 & 1890246.778 & 911763.8217 & 815048.4382 \\
\hline 18 & 3002.950783 & 23965767.71 & 7320683.517 \\
\hline 19 & 1961.263909 & 1945.459122 & 1941.608824 \\
\hline 20 & 5638.838995 & 36236.62629 & 31143.98421 \\
\hline 21 & 1716825.937 & 1470818.982 & 1140601.748 \\
\hline 22 & 3551.80744 & 3464.577495 & 3414.761688 \\
\hline 23 & 2648.322643 & 2650.848828 & 2645.270911 \\
\hline 24 & 2662.889529 & 2664.03135 & 2661.797998 \\
\hline 25 & 2738.206645 & 2732.394866 & 2730.285585 \\
\hline 26 & 2784.405028 & 2786.437922 & 2782.399055 \\
\hline 27 & 4182.280784 & 3949.888038 & 3876.071383 \\
\hline 28 & 8604.909235 & 7560.618167 & 6834.144597 \\
\hline 29 & 4595.713531 & 57578.34953 & 8049.657217 \\
\hline 30 & 19464.09273 & 27855.13969 & 19708.47701 \\
\hline
\end{tabular}

Table 6: Wilcoxon signed rank test based on the result in Table 5 .

\begin{tabular}{c|c|c}
\hline & $\mathrm{R}+$ & $\mathrm{R}-$ \\
\hline 10 agents vs 50 agents & 207 & 258 \\
10 agents vs 100 agents & 274 & 191 \\
50 agents vs 100 agents & 442 & $\mathbf{2 3}$
\end{tabular}

Table 7: The mean fitness based on 50-dimension test functions

\begin{tabular}{|c|c|c|c|c|}
\hline No. & $P=1$ & $P=10$ & $P=100$ & $P=10000$ \\
\hline 1 & 4767397 & 4543595 & 4667601 & 4702013 \\
\hline 2 & 1725833 & 5617675 & 12082776 & 1764059 \\
\hline 3 & 17351 & 16283 & 18639 & 18937 \\
\hline 4 & 537 & 521 & 529 & 529 \\
\hline 5 & 520 & 520 & 520 & 520 \\
\hline 6 & 636 & 638 & 632 & 632 \\
\hline 7 & 700 & 700 & 700 & 700 \\
\hline 8 & 804 & 804 & 803 & 804 \\
\hline 9 & 1067 & 1056 & 1059 & 1061 \\
\hline 10 & 1213 & 1179 & 1171 & 1218 \\
\hline 11 & 6011 & 6063 & 6288 & 5938 \\
\hline 12 & 1200 & 1200 & 1200 & 1200 \\
\hline 13 & 1300 & 1300 & 1300 & 1300 \\
\hline 14 & 1400 & 1400 & 1400 & 1400 \\
\hline 15 & 1549 & 1548 & 1546 & 1551 \\
\hline 16 & 1618 & 1619 & 1619 & 1619 \\
\hline 17 & 921673 & 881715 & 792874 & 942630 \\
\hline 18 & 7279155 & 6879170 & 7985422 & 3360283 \\
\hline 19 & 1942 & 1946 & 1946 & 1949 \\
\hline 20 & 31074 & 34361 & 31820 & 31710 \\
\hline 21 & 1072313 & 1246738 & 1280575 & 1215168 \\
\hline 22 & 3447 & 3392 & 3358 & 3348 \\
\hline 23 & 2645 & 2645 & 2646 & 2645 \\
\hline 24 & 2663 & 2661 & 2665 & 2662 \\
\hline 25 & 2730 & 2730 & 2730 & 2730 \\
\hline 26 & 2778 & 2778 & 2788 & 2784 \\
\hline 27 & 3890 & 3858 & 3889 & 3892 \\
\hline 28 & 6907 & 7199 & 7095 & 6920 \\
\hline 29 & 12651 & 12599 & 5492 & 6212 \\
\hline 30 & 19032 & 19734 & 19928 & 18915 \\
\hline
\end{tabular}

Table 8: Wilcoxon signed rank test based on the result in Table 7.

\begin{tabular}{ccc}
\hline & $\mathrm{R}+$ & $\mathrm{R}-$ \\
\hline 1 vs 10 & 275 & 190 \\
1 vs 100 & 194 & 271 \\
1 vs 1000 & 216 & 249
\end{tabular}

$\begin{array}{ccc}10 \text { vs } 100 & 178 & 287 \\ 10 \text { vs } 1000 & 237 & 228 \\ 100 \text { vs } 1000 & 262 & 203\end{array}$

Table 9: The mean fitness based on 10, 100, and 1000 agents. The number of iterations is 100 and the number of dimensions is 50 .

\begin{tabular}{crrrr}
\hline No. & 10 agents & 100 agents & \multicolumn{1}{c}{1000 agents } \\
\hline 1 & 1338145210 & 327605271 & 116205335 \\
2 & 79184268726 & 24854725947 & 8938780098 \\
3 & 235022.9 & 101045.4 & 43799.9 \\
4 & 17994.29 & 4197.986 & 1711.866 \\
5 & 521.2865 & 521.1728 & 521.0734 \\
6 & 667.432 & 658.1857 & 642.7808 \\
7 & 1587.334 & 2781.969 & 725.332 \\
8 & 1315.766 & 1161.581 & 1090.504 \\
9 & 1526.552 & 1359.62 & 1267.708 \\
10 & 12887.08 & 10302.09 & 8891.271 \\
11 & 14446.95 & 12404.72 & 11200.69 \\
12 & 1204.309 & 1202.841 & 1202.375 \\
13 & 1305.891 & 1303.342 & 1300.934 \\
14 & 1589.182 & 1461.033 & 1420.469 \\
15 & 1403735 & 24170.85 & 2578.101 \\
16 & 1623.134 & 1622.352 & 1621.448 \\
17 & 225197269 & 35122256 & 7038724 \\
18 & 6262617231 & 268449390 & 9439867 \\
19 & 2652.459 & 2054.65 & 1994.275 \\
20 & 373457.8 & 42918.45 & 14015.08 \\
21 & 58530680 & 8650934 & 4298820 \\
22 & 44469.24 & 3944.376 & 3346.909 \\
23 & 3411.079 & 2813.781 & 2702.812 \\
24 & 2813.254 & 2734.035 & 2708.263 \\
25 & 2813.406 & 2757.566 & 2741.351 \\
26 & 2847.602 & 2787.787 & 2754.411 \\
27 & 4932.037 & 4404.915 & 4089.907 \\
28 & 16155.92 & 11652.09 & 8520.319 \\
29 & 907086143 & 96761146 & 7618629 \\
30 & 11357918 & 1580825 & 339916.2
\end{tabular}

Table 10: Wilcoxon signed rank test based on the result in Table 9.

\begin{tabular}{c|c|c}
\hline & $\mathrm{R}+$ & $\mathrm{R}-$ \\
\hline 10 vs 100 & 450 & $\mathbf{1 5}$ \\
10 vs 1000 & 465 & $\mathbf{0}$ \\
100 vs 1000 & 465 & $\mathbf{0}$
\end{tabular}

\section{References}

[1] Muhammad B, Mohammed SK, Pebrianti D, Abdul Ghani N, Abdul Aziz NH, Ab. Aziz NA, Shapiai MI, Subari N, Ab Rahman T, Mohd Azmi KZ \& Ibrahim Z, A Black Hole Algorithm with White Hole Operator and Its Application in Parameter Tuning of Proportional-Integral-Derivative Controller, SICE International Symposium on Control Systems 2018, March 9-11, Tokyo, Japan, (2018).

[2] Mohammed SK, Ibrahim Z, Daniyal H \& Ab. Aziz NA, White Hole-Black Hole Algorithm, The National Conference for Postgraduate Research, Pekan, Pahang, Malaysia, (2016), pp. 824-833.

[3] Ibrahim Z, Abdul Aziz NH, Ab. Aziz NA, Razali S, Shapiai MI, Nawawi SW \& Mohamad MS (2015), A Kalman Filter Approach for Solving Unimodal Optimization Problems, ICIC Express Letters, Vol. 9, Issue 12, pp. 3415-3422.

[4] Ibrahim Z, Abdul Aziz NH, Ab. Aziz NA, Razali R \& Mohamad MS (2016), Simulated Kalman Filter: A Novel Estimation-Based Metaheuristic Optimization Algorithm, Advance Science Letters, Vol. 22, pp. 2941-2946.

[5] Kalman RE (2011), A New Approach to Linear Filtering and Prediction Problems, ASME Journal of Basic Engineering, Vol. 82, No. 1, pp. 35-45.

[6] Muhammad B, Mat Jusof MF, Shapiai MI, Adam A, Md Yusof Z, Mohd Azmi KZ, Abdul Aziz NH, Ibrahim Z \& Mokhtar N, Feature Selection using Binary Simulated Kalman Filter for Peak Classification of EEG Signals, $20188^{\text {th }}$ International Conference on Intelligent Systems, Modelling and Simulation, (2018), pp. 16.

[7] Adam A, Ibrahim Z, Mokhtar N, Shapiai MI, Mubin M \& Saad I (2016), Feature Selection using Angle Modulated Simulated Kalman Filter for Peak Classification of EEG Signals, SpringerPlus, 
Vol. 5, No. 1580

[8] Lazarus K, Noordin NH, Mat Jusof MF, Ibrahim Z, \& Abas KH (2017), Adaptive Beamforming Algorithm Based on a Simulated Kalman Filter, International Journal of Simulation: Systems, Science and Technology, Vol. 18, No. 4, pp. 10.1-10.5.

[9] Lazarus K, Noordin NH, Mohd Azmi KZ, Abd Aziz NH \& Ibrahim Z, Adaptive Beamforming Algorithm based on Generalized Opposition-based Simulated Kalman Filter, The National Conference for Postgraduate Research, Pekan, Pahang, (2016), pp. 1-9.

[10] Lazarus K, Noordin NH, Ibrahim Z, Mat Jusof MF, Mohd Faudz MA, Subari N, and Mohd Azmi KZ, An Opposition-Based Simulated Kalman Filter Algorithm for Adaptive Beamforming, IEEE International Conference on Applied System Innovation, (2017), pp. 91-94

[11] Lazarus K, Noordin NH, Ibrahim Z \& Abas KH, Adaptive Beamforming Algorithm based on Simulated Kalman Filter, Asia Mult Conference on Modelling and Simulation, (2016), pp. 19-23.

[12] Abd Aziz NH, Ab. Aziz NA, Ibrahim Z, Razali S, Abas KH, Mohamad MS, A Kalman Filter Approach to PCB Drill Path Optimization Problem, IEEE Conference on Systems, Process and Control, (2016), pp. 33-36.

[13] Abdul Aziz NH, Ibrahim Z, Ab. Aziz NA, Md Yusof Z \& Mohamad MS (2018), Single-Solution Simulated Kalman Filter Algorithm for Routing in Printed Circuit Board Drilling Process, Intelligent Manufacturing and Mechatronics, pp. 649-655.

[14] Mustapa A, Md Yusof Z, Adam A, Muhammad B \& Ibrahim Z (2018), Solving Assembly Sequence Planning using Angle Modulated Simulated Kalman Filter, IOP Conference Series: Materials, Science, and Engineering, Vol. 319, No. 012044.

[15] Md Yusof Z, Satiman SN, Mohd Azmi KZ Badaruddin Muhammad, Saifudin Razali, Zuwairie Ibrahim, Zulfakar Aspar, and Suraya Ismail, Solving Airport Gate Allocation Problem using Simulated Kalman Filter, International Conference on Knowledge Transfer, (2015), pp. 121-127.

[16] Mohd Azmi KZ, Md Yusof Z, Satiman SN, Muhammad B, Razali S, Ibrahim Z, Ab. Aziz NA \& Abd Aziz NH, Solving Airport Gate Allocation Problem Using Angle Modulated Simulated Kalman Filter, The National Conference for Postgraduate Research, Pekan, Pahang, Malaysia, (2016), pp. 875-885.

[17] Ann NQ, Pebrianti D, Bayuaji L, Daud MR, Samad R, Ibrahim Z, Hamid R \& Syafrullah M (2018), SKF-Based Image Template Matching for Distance Measurement by using Stereo Vision, Intelligent Manufacturing and Mechatronics, pp. 439-447.

[18] Ann NQ, Pebrianti D, Ibrahim Z, Mat Jusof MF, Bayuaji L, and Abdullah NRH (2018), Illumination-Invariant Image Matching Based on Simulated Kalman Filter (SKF), Journal of Telecommunication, Electronics and Computer Engineering, Vol. 10, No. 13, pp. 31-36.

[19] Muhammad B, Mohd Azmi KZ, Ibrahim Z, Mohd Faudzi AA \& Pebrianti D, Simultaneous Computation of Model Order and Parameter Estimation for System Identification Based on Opposition-Based Simulated Kalman Filter, SICE International Symposium on Control Systems 2018, March 9-11, Tokyo, Japan, (2018), pp. 105-112.

[20] Mohd Azmi KZ, Ibrahim Z, Pebrianti D \& Mohamad MS (2017), Simultaneous Computation of Model Order and Parameter Estimation for ARX Model Based on Single and Multi Swarm Simulated Kalman Filter, Journal of Telecommunication, Electronic, and Computer Engineering, Vol. 9, No. 1-3, pp. 151-155.

[21] Muhammad B, Pebrianti D, Abdul Ghani N, Abdul Aziz NH, Ab. Aziz NA, Mohamad MS, Shapiai MI \& Ibrahim Z, An Application of Simulated Kalman Filter Optimization Algorithm for Parameter Tuning in Proportional-Integral-Derivative Controllers for Automatic Voltage Regulator System, SICE International Symposium on Control Systems 2018, March 9-11, Tokyo, Japan (2018), pp. 113-120.

[22] Abd Aziz NH, Ibrahim Z, Razali S, Bakare TA \& Ab. Aziz NA, How Important the Error Covariance in Simulated Kalman Filter? The National Conference for Postgraduate Research, Pekan, Pahang, (2016), pp. 315-320.

[23] Abdul Aziz NH, Ab. Aziz NA, Mat Jusof MF, Razali S, Ibrahim Z, Adam A \& Shapiai MI, An analysis on the Number of Agents Towards the Performance of the Simulated Kalman Filter Optimizer, $20188^{\text {th }}$ International Conference on Intelligent Systems, Modelling and Simulation, (2018), pp. 16-21.

[24] Abdul Aziz NH, Ibrahim Z, Ab. Aziz NA \& Razali S (2017), Parameter-less Simulated Kalman Filter, International Journal of
Software Engineering and Computer Systems, Vol. 3, pp. 129-137.

[25] Abd Aziz NH, Ab. Aziz NA, Ibrahim Z, Razali S, Mat Jusof MF, Abas KH, Mohamad MS \& Mokhtar N, Simulated Kalman Filter with Randomized Q and R Parameters, International Conference on Artificial Life and Robotics, (2017) pp. 711-714.

[26] Muhammad B, Ibrahim Z, Mat Jusof MF, Ab Aziz NA, Abd Aziz NH \& Mokhtar N, A Hybrid Simulated Kalman Filter - Gravitational Search Algorithm (SKF-GSA), International Conference on Artificial Life and Robotics, (2017), pp. 707-710.

[27] Muhammad B, Ibrahim I, Mohd Azmi KZ, Abas KH, Ab. Aziz NA, Abd Aziz NH \& Mohamad MS, Performance Evaluation of Hybrid SKF Algorithms: Hybrid SKF-PSO and Hybrid SKF-GSA The National Conference for Postgraduate Research, Pekan, Pahang, Malaysia, (2016), pp. 865-874.

[28] Muhammad B, Ibrahim Z, Mohd Azmi KZ, Abas KH, Ab. Aziz NA, Abd Aziz NH \& Mohamad MS, Four Different Methods to Hybrid Simulated Kalman Filter (SKF) with Particle Swarm Optimization (PSO), The National Conference for Postgraduate Research, Pekan, Pahang, Malaysia, (2016), pp. 843-853.

[29] Muhammad B, Ibrahim Z, Mohd Azmi KZ, Abas KH, Ab Aziz NA, Abd Aziz NH \& Mohamad MS, Four Different Methods to Hybrid Simulated Kalman Filter (SKF) with Gravitational Search Algorithm (GSA), The National Conference for Postgraduate Research, Pekan, Pahang, Malaysia, (2016), pp. 854-864.

[30] Muhammad B, Ibrahim Z, Ghazali KH, Mohd Azmi KZ, Ab. Aziz NA, Abd Aziz NH \& Mohamad MS (2015), A New Hybrid Simulated Kalman Filter and Particle Swarm Optimization for Continuous Numerical Optimization Problems, ARPN Journal of Engineering and Applied Sciences, Vol. 10, No. 22, pp. 1717117176.

[31] Md Yusof Z, Ibrahim I, Satiman SN, Ibrahim Z, Abd Aziz NH, \& Ab Aziz NA, BSKF: Binary Simulated Kalman Filter, Third International Conference on Artificial Intelligence, Modelling and Simulation, (2015), pp. 77-81.

[32] Md Yusof Z, Ibrahim I, Ibrahim Z, Abas KH, Ab Aziz NA, Abd Aziz NH \& Mohamad MS, Local Optimum Distance Evaluated Simulated Kalman Filter for Combinatorial Optimization Problems, The National Conference for Postgraduate Research, Pekan, Pahang, Malaysia, (2016), pp. 892-901.

[33] Md Yusof Z, Ibrahim Z, Ibrahim I, Mohd Azmi KZ, Ab. Aziz NA, Abd Aziz NH \& Mohamad MS (2016), Distance Evaluated Simulated Kalman Filter for Combinatorial Optimization Problems, ARPN Journal of Engineering and Applied Sciences, Vol. 11, No. 7, pp. 4904-4910.

[34] Md Yusof Z, Ibrahim Z, Ibrahim I, Mohd Azmi KZ, Ab. Aziz NA, Abd Aziz NH \& Mohamad MS (2016), Angle Modulated Simulated Kalman Filter Algorithm for Combinatorial Optimization Problems, ARPN Journal of Engineering and Applied Sciences, Vol. 11, No. 7, pp. 4854-4859.

[35] Muhammad B, Mohammed SK, Pebrianti D, Abdul Ghani N, Abdul Aziz NH, Ab. Aziz NA, Shapiai MI, Subari N, Ab Rahman T, Mohd Azmi KZ \& Ibrahim Z (2018), A Black Hole Algorithm with White Hole Operator and Its Application in Parameter Tuning of Proportional-Integral-Derivative Controller, SICE International Symposium on Control Systems.

[36] Hatamlou A (2013), Black Hole: A New Heuristic Optimization Approach for Data Clustering, Information Sciences, Vol. 222, pp 175-184.

[37] Liang JJ, Qu BY \& Suganthan PN (2013), Problem Definitions and Evaluation Criteria for the CEC 2014 Special Session and Competition on Single Objective Real-Parameter Numerical Optimization.

[38] Derrac J, Garcia S, Molina D \& Herrera F (2011), A Practical Tutorial on the Use of Nonparametric Statistical Tests as a Methodology for Comparing Evolutionary and Swarm Intelligence Algorithms, Swarm and Evolutionary Computation, Vol. 1, No. 1, pp. 3-18.

[39] Ab Rahman T, Ibrahim Z, Ab. Aziz NA, Zhao S \& Abdul Aziz NH (2018), Single-Agent Finite Impulse Response Optimizer for Numerical Optimization Problems, IEEE Access, Vol. 6, pp. 9358-9374. 